\author{
Lupan Anamaria \\ CELBLF, Université Babes Bolyai, \\ Cluj-Napoca, Roumanie \\ CELIS, Université Clermont-Auvergne, \\ Clermont-Ferrand, France
}

\title{
Représentations de l'eau dans les essais yourcenariens
}

\begin{abstract}
Adept of metamorphosis, Marguerite Yourcenar repeatedly uses in her essays the image of water, fluid elements related to the idea of transformation and rebirth; sea, ocean, artificial fountain - water takes various aspects and forms; from motif in the analyzed paintings to the biological functions it exercises, water crosses the yourcenarian essays. Established in the United States of America after the Second World War, did the writer study the ecology lesson testified by her texts about water? Or do these writings rather go beyond the concrete and circumstantial level of existence to talk about a global philosophy of life, as it seems to acknowledge the title of her trilogy: As the Water That Flows?

Keywords: essays; Yourcenar; water
\end{abstract}

La cosmogonie yourcenarienne situe l'eau au premier plan; dans la bonne tradition de la mythologie, cet élément est associé aux divers personnages : la femme, la lune, les poissons. Toutefois, l'eau dépasse le niveau scriptural et devient plutôt, chez l'écrivaine, une réelle inquiétude, une vive préoccupation; dans ses textes écocritiques sur l'eau, Marguerite Yourcenar continue à montrer sa passion pour la nature ; ces écrits font preuve de ce que Bruno Blanckeman appelle 
«l'humanisme participatif » ${ }^{1}$ (Blanckeman $2017:$ 169), c'est-à-dire la mise en place d'un réseau entre l'homme et les autres aspects de l'univers. Dans quel but l'écrivaine recourt à l'image de l'eau ? Veutelle être fidèle à une certaine philosophie ou sa poétique fluide, métamorphique, se plie bien à la virtualité de l'eau ? Afin de répondre à cette problématique, une étude en trois étapes sera entamée. Dans un premier temps, vont être identifiés et analysés les formes et les aspects de l'eau tels qu'ils apparaissent dans les essais ; ensuite, les valences mythologiques de ces représentations vont être interrogées; et enfin, l'analyse des valeurs métaphoriques, voire philosophiques, de l'eau, nous aidera à comprendre l'importance de ce signe herméneutique dans l'écriture de Marguerite Yourcenar.

L'eau dans la poétique yourcenarienne

Marguerite Yourcenar écrit des essais pour poser des questions à la société, pour informer le public ou bien elle y cherche sa voix. La nature la préoccupe dès son jeune âge, dès son enfance à Monts Noirs. Dans ses quatre recueils d'essai, Sous bénéfice d'inventaire (1962), Le Temps, ce grand sculpteur (1983), En Pèlerin et en étranger (1989) et Le Tour de la prison (1991), beaucoup de textes traitent de la nature et de l'eau. D'une présence artistique ou décorative, celle-ci arrive à questionner l'état de notre Terre.

L'eau est un topos de la poétique yourcenarienne ; celle-ci apparaît dans l'univers pictural, dans les tableaux qu'elle aborde en tant qu'essayiste, aussi bien que dans les décors qu'elle décrit et dans la présentation du corps humain, dans l'homme lui-même. Tableaux, nature et corps humain - tous sont traversés par l'eau; comme si l'écrivaine rassemblait à l'aide de ce terme commun, l'eau, ses centres

\footnotetext{
${ }^{1}$ Dans le Dictionnaire Marguerite Yourcenar dirigé et préfacé par Bruno Blanckeman (Paris, Champion, 2017), l'auteur propose la notion d' " humanisme participatif » pour définir le passage de Marguerite Yourcenar du «nageur à la vague »; ce concept explique la sortie de l'écrivaine de sa tour d'ivoire; elle quitte le monde livresque et commence à voir dans l'écrit un cri. De plus, elle se rend compte de l'interdépendance de la vie; le centre disparait: l'humain, le végétal et l'animal vivent dans une harmonie totale.
} 
d'intérêt : l'art, l'écologie et l'humanité. La question qui se pose c'est de savoir quelles fonctions offre chacun de ces lieux à l'eau et, d'autre part, quelle forme revêt celle-ci dans chaque endroit.

Critique impressionniste d'art, Marguerite Yourcenar écrit des essais sur la peinture ${ }^{2}$ des divers pays et périodes; Albrecht Dürer $\left(\mathrm{XV}^{\mathrm{e}}\right.$ siècle, Allemagne), Michel-Ange ( $\mathrm{XVI}^{\mathrm{e}}$ siècle, Italie), Nicolas Poussin (XVII ${ }^{\mathrm{e}}$, France), Arnold Böcklin (fin du XIX ${ }^{\mathrm{e}}$ siècle, Suisse) et Rembrandt Harmenszoon van Rijn (XVII ${ }^{\mathrm{e}}$ siècle, Hollande) sont les figures les plus représentatives de sa critique artistique.

Dans chaque analyse, le motif de l'eau est identifié et examiné ; il est en étroite relation aux couleurs, à la chromatique, trait emblématique de la poétique yourcenarienne. Le caractère visuel de l'écriture s'y rend visible : la couleur est accompagnée par un examen de la lumière. Par exemple l'essai sur la peinture de Poussin parle de l'eau noire tandis que celui sur Dürer de l'eau limpide et sombre.

Chaque peintre a sa spécificité ; cependant, pour garder la richesse de l'élément aquatique, les peintres utilisent, à tour de rôle, dans leurs tableaux tous les aspects de l'eau. Le devenir de l'eau est condensé dans chaque analyse : l'eau-miroir apparait à côté de l'eau stable. La diversité des images de l'eau présentes dans les critiques d'art yourcenariennes rappelle un autre trait emblématique de son écriture : la volonté de tout dire, la volonté d'exhaustivité. Poussin emploie cette métamorphose de l'eau dans ses paysages où sont illustrés 《golfes, étangs, vases $»^{3}$ (Yourcenar 1991: 471) et Dürer la fait figurer dans ses gravures; un aspect attire l'attention de la critique : chez les deux, l'eau est sereine, limpide et n'a pas de forces de destruction ; c'est plutôt une eau «qui tend à l'équilibre » (Yourcenar 1991 : 471). Partie du milieu naturel, l'eau suggère, dans les tableaux,

\footnotetext{
${ }^{2}$ Dans le recueil Le Temps, ce grand sculpteur, on trouve l'analyse d'un rêve de Durer, «Sur un rêve de Durer »; En pèlerin et en étranger présente l'essai sur la peinture française, "Une exposition Poussin à New York", sur celle allemande, «L'île des Morts de Böcklin » et sur celle hollandaise, « Deux noirs de Rembrandt ».

3 Marguerite Yourcenar, Essais et mémoires, avant propos de l'éditeur, Paris, Gallimard, coll. «Bibliothèque de la Pléiade », 1991, p. 471. Désormais on appelle cet ouvrage par l'abréviation $E M$.
} 
l'indistinction entre réel et rêve : en se faisant miroir, par son aspect réfléchissant, l'eau questionne la différence entre l'imagination et l'objectivité, ou, comme dirait Marguerite Yourcenar, elle, l'eau, fait appel aux « Jeux de miroirs et feux follets».

Les cadres construits autour de l'eau peuvent être soit artistiques, et donc, des représentations, soit des contextes concrets offerts par le milieu naturel ou construits par la société qui se veut moderne et progressive.

La nature se réjouit d'une eau libre, naturelle ; toutefois, le progrès lui impose un certain rythme : la fontaine, eau artificielle, esquisse une eau en quelque sorte domptée, parce que limitée. Les plus tristes sont les décors construits afin d'impressionner le public. Le décor du quai où se promène Oscar Wilde, le quai d'Immacolata Vecchia, est misérable ; l'écrivaine ajoute, avec une certaine ironie, que le charme de celui-ci «n'a jamais existé que sur cartes postales » (Yourcenar 1991 : 499); parfois la beauté d'un paysage est un piège, un simulacre ; afin de poétiser le réel, la société embellit artificiellement le cadre. C'est pour lutter contre ce mensonge que Marguerite Yourcenar voit dans l'écrit un cri, un témoignage.

Les constructions modernes limitent la liberté de l'eau ; celle-ci y est obligée de suivre un certain trajet. Les jets d'eau des fontaines artificielles transforment l'eau en flamme; toutefois, celle-ci retrouve sa liberté en descendant (Yourcenar 1991: 406) ; l'eau n'y renonce pas à sa beauté : elle s'adapte tout en se métamorphosant en flamme.

Le vrai mal que l'homme impose à l'eau et à la terre, c'est l'indifférence. Par la pollution, l'homme montre qu'il n'est pas responsable; il se rapproche de l'inhumain tout en s'éloignant de la nature, son essence. L'océan devient un lieu martyr où on sacrifie les poissons : « nos océans où le poisson est sacrifié aux pétroliers » (Yourcenar 1991 : 371) ; il y a une sorte de religiosité de l'eau : elle est le cadre des sacrifices. Si, généralement, dans la mythologie, l'eau avait des forces dévastatrices, dans la société moderne l'eau nuit parce qu'elle est, à son tour, tuée, polluée. En dépit du traitement cruel que les gens imposent à l'eau, celle-ci continue le plus souvent à construire la nature ; élément actif et participatif, elle érode les roches 
et collabore avec la terre à la mise en place d'un nouveau relief. Encore une fois, l'eau fait preuve de ses capacités de se métamorphoser.

L'alchimie vitale commence par l'eau: «Ton corps aux trois quarts composé d'eau, plus un peu de minéraux terrestres, petite poignée » (Yourcenar 1991 : 407) nous dit Marguerite Yourcenar; l'eau définit l'être humain. En fait, il est construit par la nature : par le feu qui l'anime et par la terre qui lui donne forme. Un lien indispensable existe, par conséquent, entre l'homme et le monde. La position centrale que l'homme avait occupée tant de temps disparaît ; il devient une «petite poignée », un élément parmi d'autres. Dans les années 1960 l'écrivaine commence à parler d'une «éthique de l'environnement non anthropocentrée » (Sanz 2000 : 174), selon la notion forgée par Teofilo Sanz; dorénavant, l'objectif essentiel ne sera plus d'assurer un lieu essentiel à l'homme, mais d'améliorer tant que possible la vie; c'est pour y parvenir que l'écrivaine essaie de réveiller la conscience des gens et de leur ouvrir les yeux tout en leur présentant des images répugnantes de leur société «à cloisons étanches ${ }^{4}$.

L'eau est partout: dans l'art, dans la nature et dans notre corps, elle travaille et offre une leçon de métamorphose par ses multiples formes et aspects. L'eau est sans forme ; c'est pour cela aussi qu'elle est si surprenante: elle change sans cesse. Cette capacité métamorphique de l'eau est très bien illustrée par Marguerite Yourcenar; dans ses essais, l'eau statique côtoie l'eau-miroir, et l'eau close et lisse juxtapose l'eau artificielle. L'eau est une richesse en soi : elle a la force de l'adaptation.

L'eau est un spectacle en soi; elle a la force de modifier un paysage, de lui donner vie et de le rendre à chaque fois visible. Voyageuse intrépide, Marguerite Yourcenar situe parmi «les plus

\footnotetext{
${ }^{4}$ Ce syntagme reprend le titre d'un essai de Marguerite Yourcenar, «Une civilisation à cloisons étanches »; dans ce texte, il s'agit de la violence faite aux animaux dans les abattoirs; pour cacher la manière inhumaine dont sont traités les animaux ces bâtiments sont éloignés de la ville et de la vue des gens.
} 
saisissants » (Yourcenar 1991: 406) tableaux ceux avec des «fjords de l'Alaska et de la Norvège », où on rencontre l' « eau frissonnante, mais étale, du fjord, eau ruisselante des cascades [...], vapeur qui s'élève de leur chute, eau qui [...] fait route au ciel, gel et neige $[\ldots] »$; comme réfléchie dans le miroir florentin de Zénon ${ }^{5}$, l'eau prend plusieurs formes.

La vraie réussite de l'eau est d'être détachée - telle apparaît celleci dans l'essai sur Oscar Wilde: "l'indifférente mer qui ne sait pas qu'elle est bleue » (Yourcenar 1991: 506) ; ce détachement rend la mer vraiment libre et, par d'autre part, propose, en filigrane une leçon à la société trop limitée par les modes ou par divers compromis ; d'ailleurs, dans ses pensées de Sources $I I^{6}$, on trouve les conseils que l'écrivaine offrait à un jeune; elle lui suggère de ne jamais se laisser forger par l'extérieur, de ne jamais s'attacher. C'est cette même leçon que donne la mer : elle sait qu'elle est mais elle ne s'intéresse pas à son aspect. Elle est « informe » (Yourcenar $1991: 618)$.

L'eau forme et construit la société ; présente partout, elle enseigne la liberté, la puissance de l'adaptation et le détachement. Maître authentique, elle parle de l'héritage universel et essaie de rendre conscients les gens de leur responsabilité envers leur origine.

Eau et mythologie

Esprit curieux, Marguerite Yourcenar ne veut pas que son intérêt pour l'eau reste au niveau personnel, subjectif ; l'écrivaine s'informe sur ce sujet, comme le prouvent les livres de sa bibliothèque de Petite Plaisance. Dans la bibliothèque de la salle à manger, dans la partie derrière la porte ouvrant sur l'entrée, on trouve l'étude Histoire géologique de la Mer de Stanislas Meunier (Bernier 2004 : 45) et la revue minéralogique (Bernier 2004 : 51) Le Monde et les minéraux;

\footnotetext{
${ }^{5}$ Personnage yourcenarien du roman L'CEuvre au noir paru en 1968.

${ }^{6}$ Elle dit à tous ceux qui lui demandent l'avis sur la vie: " $\mathrm{Ne}$ t'attache pas. $\mathrm{Ne}$ t'attache jamais. »; Marguerite Yourcenar, Sources II, éd. Élyane Dezon-Jones, Paris, Gallimard, «Cahiers de la NRF », 1999, p. 249.
} 
l'inventaire d'Yvon Bernier compte bien d'autres études sur la chimie et la nature.

L'écrivaine se forge une mythologie personnelle de l'eau; elle voit que dans les interprétations des peintres qu'elle analyse, Poussin et Dürer, le « déluge de type biblique » (Yourcenar 1991 : 371) n'est pas présent. Elle ne souscrit plus aux valences dévastatrices de l'eau ; tout au plus, l'eau apporte l'oubli parce qu'elle noie et fait disparaitre des personnes et des objets. Ceci se passe dans l'essai «L'Italienne à Alger» où «une énorme lame de fond» (Yourcenar 1991: 618) anéantit une femme avec «le chapeau du grand magasin, le manteau, le sac, les papiers d'identité avec les portraits des enfants ou des petits-enfants, en fait, toute une vie » (Yourcenar 1991: 618); l'eau efface toutes les traces. Ce n'est que la mémoire qui peut refaire les décors d'avant les passages de l'eau; dans l'essai avec la femme prise par la mer, l'écrivaine s'imagine être « la seule personne sur la terre » (Yourcenar 1991 : 618) à se souvenir d'elle. Par conséquent, l'eau ne fait pas une catastrophe : elle efforce plutôt les gens à se rappeler le passé.

La responsabilité de la société envers l'eau transparait aussi ses essais écocritiques; ces textes montrent encore une fois son intérêt pour l'environnement; ses prises de position contre la pollution mettent en scène sa volonté d'agir concrètement. La mythologie de la responsabilité de l'homme y fait jour; selon l'écrivaine, le mal fait à la terre habitue l'homme à la violence; en fin de compte, il va accepter plus facilement le mal envers son prochain, une fois habitué au mal envers les autres éléments de l'univers.

L'eau parle de la liberté aussi parce qu'elle invite au voyage, à la rencontre des choses nouvelles. Dans «L'Air et l'Eau éternels », est formulée la beauté de l'évasion à l'aide de l'eau : «Le navire est beau comme tous les navires en partance» (Yourcenar 1991: 622). Marguerite Yourcenar n'est pas une traditionaliste aveugle, mais elle invite à un équilibre dans tout; le voyage en navire devient une sorte $\mathrm{d}$ 'art ; celui doit respecter quelques règles afin de ne pas endommager le rythme de l'univers. Il doit se faire sans vitesse ; cette activité n'est pas utilitaire, mais elle s'adresse « à cette bizarre classe de vagabonds 
riches » (Yourcenar 1991: 622). Afin de rendre l'eau éternelle, les gens doivent se faire des artistes de leur propre vie: ils doivent considérer les éléments de la nature comme un trésor à conserver.

D'autre part, l'eau devient une œuvre d'art sous la main de Marguerite Yourcenar; elle emprunte l'image de l'eau pour donner le titre à deux de ses œuvres : Fleuve profond, sombre rivière et Comme l'eau qui coule. Le premier représente un recueil de traduction de negro spirituals publié afin de donner voix à la souffrance des esclaves d'Amérique; le deuxième est un recueil de trois nouvelles paru en 1982: Anna, soror..., Un homme obscur et Une belle matinée. La métaphore de l'eau est employée à plusieurs reprises pour suggérer la rapidité ; par exemple, dans le texte sur Mozart, les notes «coulant comme l'eau » (Yourcenar 1991: 484) intègrent la musique dans le processus dynamique de la vie. L'eau coule vite, indifférente, comme la vie ; la fugacité du temps s'inscrit dans la philosophie héraclitienne mais l'écrivaine n'adhère pas en totalité à cette pensée; elle s'intéresse plutôt, comme on l'a vu déjà, à une mythologie de l'eau, aux leçons que l'élément liquide peut donner à la société.

La société emploie l'eau dans son langage courant, mais aussi dans le «balbutiement » d'après les grands conflits mondiaux. Les métaphores recourent à l'image de l'eau afin de suggérer le passage fulgurant de la vie ou la catastrophe; la période d'aguerrissement d'après la deuxième Guerre Mondiale impose l'utilisation d'un langage plus plastique pour poétiser la cruauté du réel. Cependant, Marguerite Yourcenar est contre l'emploie des figures comme «vagues » ou «plage balnéaire inondée » dans la présentation de la vie de septembre 1944 ; et cela parce qu'elle considère que les vagues ont un rythme invariable, rythme imposé par le dieu des flots : elles avancent et, puis, elles reculent. Pour parer aux désastres, il faut que les hommes agissent; ils doivent être responsables s'ils veulent être

7 Dans la «Chronologie» d'Euvres romanesques (Paris, Gallimard, coll. «Bibliothèque de la Pléiade », 1982, p. XXIII), Marguerite Yourcenar explique la genèse de ces poèmes : « entreprises en guise de délassement ou d'exercices ». 
libres. Elle critique de même la manière de voir les "vagues de l'avenir » comme un bien inexorable.

Les métaphores ne doivent pas faire oublier aux gens le processus de la vie; elles ne doivent pas devenir des masques pour la réalité parce qu'à ce moment elles introduisent la maladie tout en empêchant les hommes de bien voir, comme cela se passe dans le cas des habitudes.

L'eau a des qualités qui la rendent vivante; son dynamisme, l'assimile à la vie toujours en mouvement: «Toute eau aspire à devenir vapeur, et toute vapeur à redevenir eau » (Yourcenar 1991 : 406); les modifications de son état nous rappellent le devenir d'un jeune homme. De plus, l'eau s'adapte; une image surprenante indique la religiosité de cet élément: "L'eau, qui d'elle-même cède et descend. Et c'est pourquoi lui convient le qualificatif franciscain: umile » (Yourcenar 1991: 405) ; elle connaît sa place dans l'univers et réussit à rallier ses fonctions à ses désires et, par conséquent, elle devient libre; plus qu'une composante du corps humain, l'eau présente, dans les essais yourcenariens, des valences humaines ou même elle arrive à donner des leçons aux êtres humains.

Apprendre la société à cohabiter - tel est le désir de Marguerite Yourcenar ; apprendre des autres, connaître son héritage et être libre et responsable. Dans sa liste des «Haines » de Sources II, Marguerite Yourcenar note l'écart entre l'homme et l'univers : «La séparation de l'homme d'avec les formes animales et végétales vivantes » (Élyane 1999 : 242); elle essaie de transmettre, dans ses écrits, une leçon d'harmonie, où tout inspire l'humanité.

L'eau-même est humaine parce qu'elle est dynamique et parce qu'elle participe activement à la vie et à sa métamorphose.

Conclusion

La leçon yourcenarienne de l'eau est une leçon de liberté ; elle nous apprend la mythologie de la responsabilité où, sans rester le centre de l'univers, l'homme participe activement à la beauté de la vie. Et puis, l'eau nous apprend le détachement, la capacité de ne jamais nous attacher afin de voir toujours clairement, en ensemble, les choses. En 
fin, l'eau invite à l'étonnement, au miracle de la vie : c'est elle qui assure, par la diversité de ses formes, un autre paysage et nous invite à aller toujours vers l'inconnu.

\section{Bibliographie}

Bernier, Y. (2004) : Inventaire de la bibliothèque de Marguerite Yourcenar - PetitePlaisance. Clermont-Ferrand : SIEY.

Blanckeman, B. (2017) : Eau. In : B. Blanckeman (éd.), Dictionnaire Marguerite Yourcenar. Paris : Honoré Champion.

Élyane, D.-J. (1999) : Sources II. Paris : Gallimard, « Cahiers de la NRF ».

Sanz, T. (2000) : Aspects de l'horizon éthique et esthétique de Marguerite Yourcenar : sa lecture des Tragiques d'Agrippa d'Aubigné. In : C. Biondi, F. Bonali-Fiquet, M. Cavazutti, E. Pessini (éd.), Marguerite Yourcenar. Parcours, méthodes et finalités d'une écriture critique, actes du colloque de Modène, Parme et Bologne, mai 1999. Tours : SIEY.

Yourcenar, M. (1991): Essais et mémoires, avant propos de l'éditeur. Paris : Gallimard, coll. «Bibliothèque de la Pléiade».

Yourcenar, M. (1982) : Euvres romanesques. Paris : Gallimard, coll. «Bibliothèque de la Pléiade ». 